\title{
Possible signals of two QCD phase transitions at NICA- FAIR energies
}

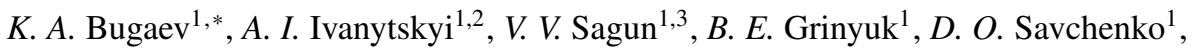

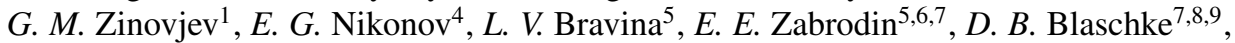 \\ S. Kabana ${ }^{10,11}$, and A. V. Taranenko ${ }^{7}$
}

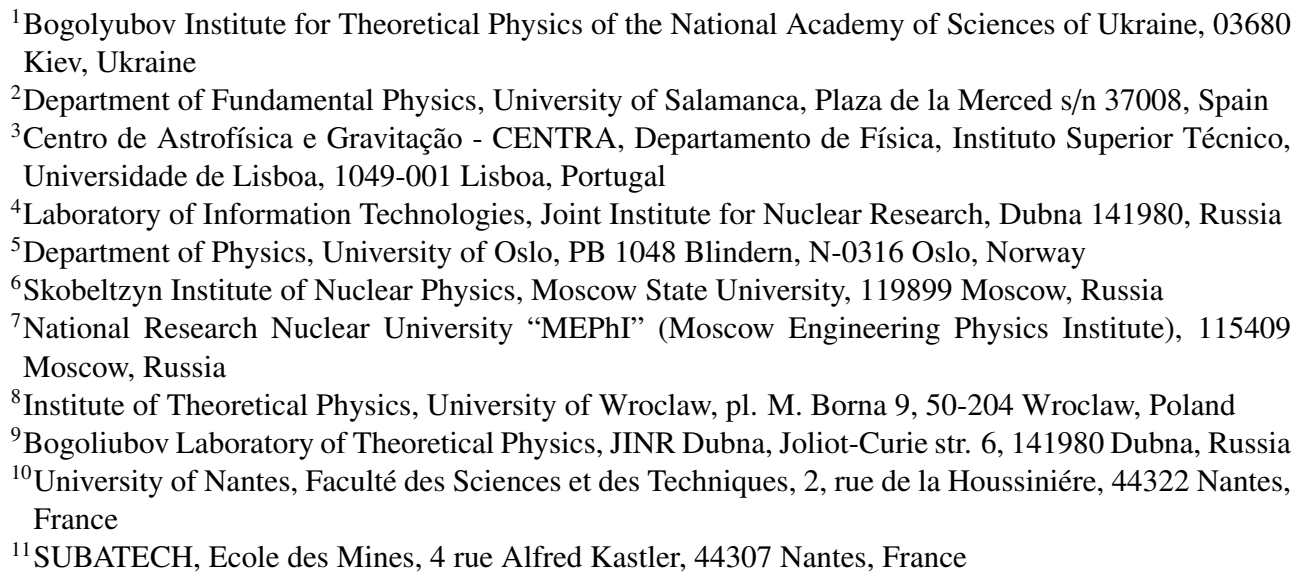

Abstract. The chemical freeze-out irregularities found with the most advanced hadron resonance gas model and possible signals of two QCD phase transitions are discussed. We have found that the center-of-mass collision energy range of tricritical endpoint of QCD phase diagram is $[9 ; 9.2] \mathrm{GeV}$ which is consistent both with the QCD inspired exactly solvable model and experimental findings.

\section{Introduction}

The experimental programs of heavy ion collisions planned at RHIC (BNL) [1], NICA (JINR) [2] and FAIR (GSI) [3] are aimed to study the phase diagram of strongly interacting matter. The major tasks of these experiments are to determine the threshold collision energies of two phase transitions (PTs) expected to exist in quantum chromodynamics (QCD) and to locate their tricritical (or two critical endpoints). However, until recently the situation with the reliable signals of the chiral symmetry restoration (CSR) and the deconfinement PTs was not clear. Thus, it was not clear whether at high baryonic densities there are two different phase transitions or a single one. Furthermore, neither the order of these transitions nor their order were clear until recently.

Although some promising irregularities in the behavior of experimental data, known in the literature as the Kink [4], the Strangeness Horn [5] and the Step [6], were found, their relation to any of two QCD phase transitions remains unclear. There are two problems with the

\footnotetext{
*e-mail: Bugaev@th.physik.uni-frankfurt.de
} 
theoretical model with which the collision energy dependence of $K^{+} / \pi^{+}$ratio was predicted [5]: first, at high baryonic densities the deconfinement PT occurs in this model at temperature above $200 \mathrm{MeV}$, which simply contradicts to the present lattice QCD findings; second, within the model of [5] the number of non-strange degrees of freedom is 16 and the number of strange degrees of freedom is 14 , which means that the total number of degrees of freedom is 30 that is essentially below the number of degrees of freedom of QCD with 3 quark flavors and 3 colors. Hence, we consider the model of [5] as a successful parameterization of experimental data, but one cannot draw reliable physical conclusions from it.

At the same time during last few years two theoretical groups, namely the Kiev and the Giessen ones, reported the signals of two QCD PTs, and, remarkably, they independently concluded [7-12] that the CSR PT occurs in the hadronic phase at the center-of-mass collision energy $\sqrt{s_{N N}} \simeq 4-5 \mathrm{GeV}$, while the deconfinement PT to quark gluon plasma (QGP) occurs at the collision energy $\sqrt{s_{N N}} \simeq 9-10 \mathrm{GeV}$. Furthermore, both groups argued that the CSR is the first order PT [10-12], while the deconfinement PT has a second order [10-13] or a weak first order [12]. Moreover, combining the results of [14] on the enhancement of light nuclei fluctuations at $\sqrt{s_{N N}} \simeq 8.8 \mathrm{GeV}$ with the hypothesis of existence of two QCD PTs, we have come to a conclusion that the collision energy range $\sqrt{s_{N N}} \simeq 8.8-9.2 \mathrm{GeV}$ corresponds not to a vicinity of CEP as it was suggested in [14], but to a vicinity of 3CEP [12, 15].

Due to importance of these findings for the planned experimental programs, here we would like to discuss them in some details and reinforce them with the new arguments. The work is organized as follows: in Sect. 2 the main results of the multicomponent hadron resonance gas model (HRGM) are presented; while Sect. 3 is devoted to a discussion of two QCD PTs; our conclusions are summarized in Sect. 4.

\section{HRGM with Multicomponent Hard-Core Repulsion}

The possible PTs signals mentioned above were obtained by Kiev group using the multicomponent HRGM (MHRGM) [7-9, 12, 15-21] which has 4 or 5 hard-core radii of hadrons. In contrast to the HRGM with one or two hard core radii of hadrons [22], the MHRGM2 of $[20,21]$ has the following hard-core radii of pions $R_{\pi}=0.15 \mathrm{fm}$, kaons $R_{K}=0.395 \mathrm{fm}, \Lambda$ hyperons $R_{\Lambda}=0.085 \mathrm{fm}$, other baryons $R_{b}=0.365 \mathrm{fm}$ and other mesons $R_{m}=0.42 \mathrm{fm}$. Having only 2 or 3 additional global fitting parameters compared to the usual HRGM [22], one can achieve an extremely good description of the hadronic multiplicity ratios measured at AGS, SPS, RHIC and LHC energies with a high quality $\chi^{2} / d o f \simeq 1.08[20,21]$, including traditionally the most problematic ones for the usual HRGM [22], i.e. $K^{+} / \pi^{+}, \Lambda / \pi^{+}$and $\bar{\Lambda} / \pi^{-}$ ratios. The most successful MHRGM (MHRGM1) based on the Van der Waals approximation is developed in [19] with the hard-core radii of baryons $R_{b}=0.355 \mathrm{fm}$, mesons $R_{m}=0.4$ fm, pions $R_{\pi}=0.1 \mathrm{fm}$, kaons $R_{K}=0.38 \mathrm{fm}$, and $\Lambda$ hyperons $R_{\Lambda}=0.11 \mathrm{fm}$ which provide a similar fit quality as the MHRGM2.

A high quality fit of hadronic multiplicity ratios achieved by the MHRGM gives us a high confidence that the equation of state of hadronic matter is now fixed with high accuracy in the wide range of chemical freeze-out (CFO) temperature $T$ and baryonic chemical potential $\mu_{B}$, since the MHRGM2 [20,21] allows one to go beyond the Van der Waals approximation traditionally used in HRGM.

More details on the MHRGM and the used fitting procedure can be found in [15, 1921]. Some results obtained within the MHRGM1 and MHRGM2 are shown in Figs. 1 and 2. From the left panel of Fig. 1 one can see that the CFO temperature strongly jumps, when the collision energy increases from $\sqrt{s_{N N}} \simeq 4.3 \mathrm{GeV}$ to $\sqrt{s_{N N}} \simeq 4.9 \mathrm{GeV}$. As one can see from the left panel of Fig. 2, at this energy range the CFO pressure increases by 6 times! The right panel of Fig. 2 shows that at the collision energy $\sqrt{s_{N N}} \simeq 4.9 \mathrm{GeV}$ there is a huge 

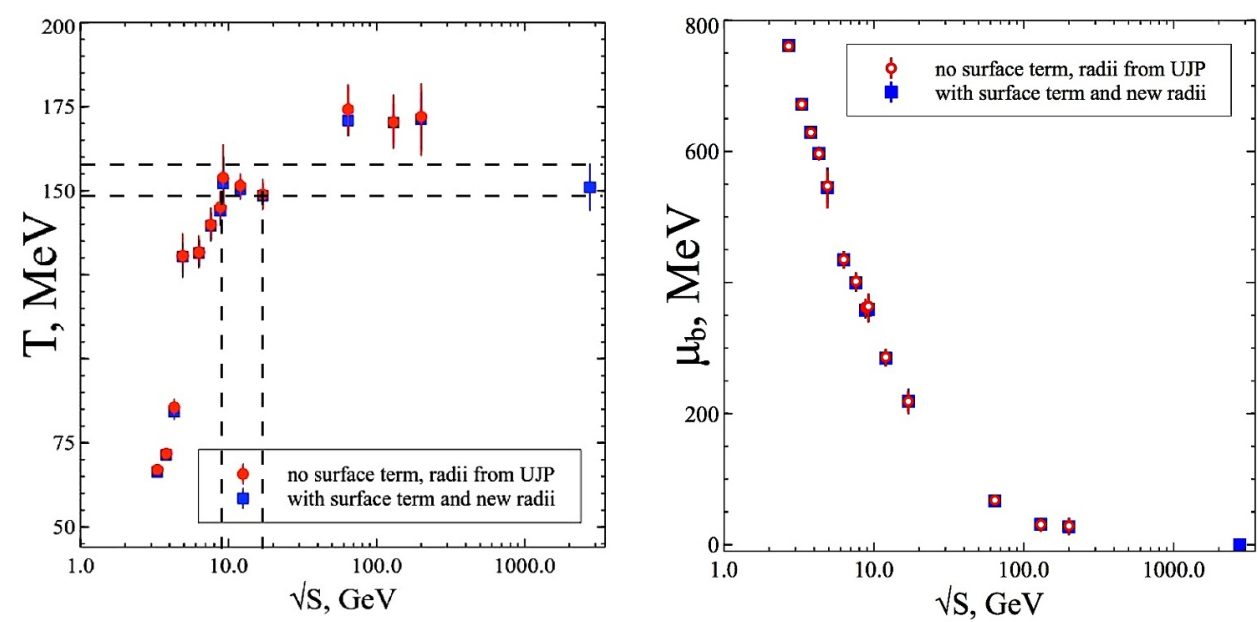

Figure 1. Left panel: The CFO temperature as a function of the collision energy found in [21] within MHRGM1 (circles) and MHRGM2 (squares). The horizontal dashed lines correspond to the range of the (3)CEP temperature values found in [23]. The vertical dashed lines indicate the corresponding values of the collision energy at which the CEP can be reached (see text for details). Right panel: The same as in the left panel, but for the baryonic chemical potential

maximum of the baryonic charge density. In other words, increasing the collision energy from $\sqrt{s_{N N}} \simeq 4.3 \mathrm{GeV}$ to $\sqrt{s_{N N}} \simeq 4.9 \mathrm{GeV}$ by $16 \%$ one can create a dense and small system with very high pressure. The question is: what kind of state is it?

Further analysis of thermodynamics at CFO performed in works [7-9, 12] revealed a few irregularities observed at CFO which are related to two QCD phase transitions. The most remarkable irregularities include two sets of correlated quasi-plateaus found in the collision energy dependence of the entropy per baryon, the total pion number per baryon, and thermal pion number per baryon [7-9]. They are located at the collision energy ranges $\sqrt{s_{N N}} \simeq$ $3.8-4.9 \mathrm{GeV}$ and $\sqrt{s_{N N}} \simeq 7.6-9.2 \mathrm{GeV}$. Also there are two peaks of trace anomaly $\delta=\frac{(\epsilon-3 p)}{T^{4}}$ (here $\epsilon, p$ and $T$ denote, respectively, the energy density of the system, its pressure and temperature) observed at the maximal energy of each set of quasi-plateaus $[9,12]$. Also it is remarkable that at CFO two sharp peaks of the baryonic charge density (see the right panel of Fig. 2) are located exactly at the collision energies of the trace anomaly peaks [12], i.e. at $\sqrt{s_{N N}}=4.9 \mathrm{GeV}$ and $\sqrt{s_{N N}}=9.2 \mathrm{GeV}$. This is of outstanding importance, since the inflection point/maximum of the trace anomaly is traditionally used in lattice QCD to determine the pseudocritical temperature of the cross-over transition [26].

Note that the set of low energy quasi-plateaus in the collision energy dependence of the entropy per baryon, the total pion number per baryon, and thermal pion number per baryon was predicted a long time ago $[24,25]$ as a manifestation of the anomalous thermodynamic properties of the mixed phase of the first order phase transition. Since each set of found quasi-plateaus is accompanied by the peak of trace anomaly and by the peak of baryonic charge density, in $[8,9,12]$ it was inferred that the high energy quasi-plateaus correspond to another PT. However, till almost nowadays the nature of the matter produced in the collision energy range $\sqrt{s_{N N}} \simeq 4.9-9.2 \mathrm{GeV}$ was unclear. 

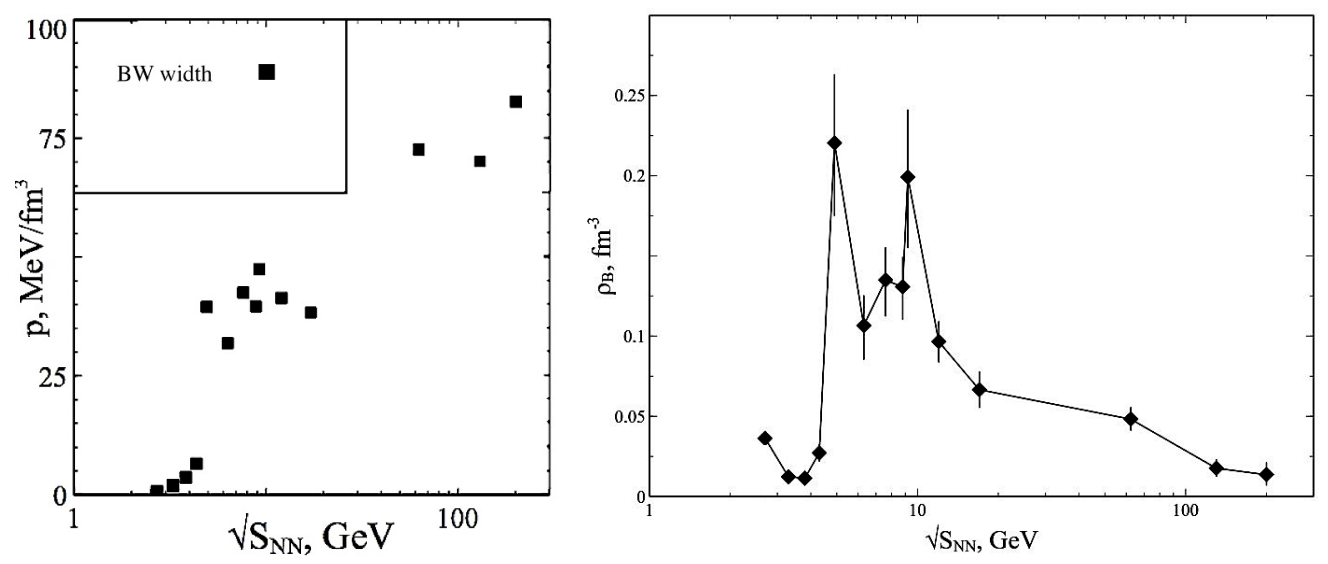

Figure 2. Left panel: Pressure at chemical freeze-out as a function of the collision energy MHRGM2 (squares) [21]. The Breit-Wigner parameterization of the resonance width is used in this model. Right panel: The same as in the left panel, but for the baryonic charge density

\section{Hadronic Matter with CSR}

Using the MHRGM1 of [7, 8] it was possible from fitting the entropy per baryon along the shock adiabat $[24,25]$ to determine the equation of state of the phase created at the collision energy range $\sqrt{s_{N N}} \simeq 4.9-9.2 \mathrm{GeV}$, i.e. between two maxima of baryonic density at CFO (see the right panel of Fig. 2). This equation of state is similar to the MIT-Bag model:

$$
p_{\text {Chiral }}=A_{0} T^{4}+A_{2} T^{2} \mu^{2}+A_{4} \mu^{4}-B,
$$

but the coefficients $A_{0} \simeq 2.53 \cdot 10^{-5} \mathrm{MeV}^{-3} \mathrm{fm}^{-3}, A_{2} \simeq 1.51 \cdot 10^{-6} \mathrm{MeV}^{-3} \mathrm{fm}^{-3}, A_{4} \simeq 1.001$. $10^{-9} \mathrm{MeV}^{-3} \mathrm{fm}^{-3}$, and $B \simeq 9488 \mathrm{MeV} \mathrm{fm}^{-3}$ are rather different from what is predicted by the perturbative QCD for massless gluons and (anti)quarks. In [12] the equation of state (1) was suggested to find out the number of bosonic and fermionic degrees of freedom of this phase. Recalling that first three terms on the right hand side of Eq. (1) correspond to the gas of massless particles and noting that the coefficient $A_{4}$ is very small and its value is comparable to its own error, we could determine the numbers of total $N_{d o f}^{t o t}$, bosonic $N_{b}^{e f f}$ and fermionic $N_{f}^{e f f}$ degrees of freedom as

$$
N_{d o f}^{t o t}=\frac{90}{\pi^{2}} A_{0} \hbar^{3} \simeq 1770, \quad N_{f}^{e f f}=12 A_{2} \hbar^{3} \simeq 141, \quad N_{b}^{\text {eff }}=N_{\text {dof }}^{\text {tot }}-\frac{7}{4} N_{f}^{\text {eff }} \simeq 1523
$$

Since the numbers $N_{b}^{e f f}$ and $N_{f}^{e f f}$ are essentially larger than the corresponding number of degrees of freedom in perturbative QCD, but at the same time $N_{d o f}^{\text {tot }}$ is of same order as the total number of spin-isospin degeneracies of all known non-strange hadrons, in [12] it was concluded that the equation of state (1) corresponds to the gas of massless hadrons with strong attraction given by the vacuum pressure $B$. Together with the results of $[10,11]$ these are the strongest arguments in favor of CSR in the hadronic phase.

It is interesting that the model of [5] also employs the equation of state (1) for the nonstrange massless degrees of freedom of the "White Matter", while the number of degrees of freedom of strange particles which have the mass of kaons is 14 . Therefore, by the construction the "White Matter" model is a mixture of massless non-strange particles and meson-like strange ones with $N_{d o f}^{t o t}=30$ [5]. Note that the number $N_{d o f}^{\text {tot }}=30$ does not fit the QCD with 3 colors and 3 quark flavors. Moreover, the deconfinement PT temperature in the model 
of [5] is above $200 \mathrm{MeV}$ which is essentially larger than the contemporary value of the QCD pseudocritical temperature $T_{p c}=156 \pm 6 \mathrm{MeV}$ found at vanishing baryonic density [26]. Therefore, it is quite possible that, if the authors of [5] used more realistic equation of state for the hadronic phase, it would lower their PT temperature, but would increase the number of degrees of freedom. Thus, the irregularities found earlier in [4-6], probably, provide the first evidence for existence of the hadronic matter with CSR, but, due to oversimplified hadronic matter description used in [5], the estimate obtained in [5] for the collision energy threshold is somewhat higher.

Now we turn to discuss the collision energy threshold of the 3CEP. As it was mentioned above the recent analysis of fluctuations of light nuclei led to a conclusion that the QCD phase diagram CEP is located in the vicinity of $\sqrt{s_{N N}} \simeq 8.8 \mathrm{GeV}[14,27]$. However, the equation of state used in $[14,27]$ contains the metastable branch (spinodal instability), which is hardly consistent with the hypothesis of local thermal equilibrium used in QCD phenomenology. Hence, we agree only with the arguments of $[14,27]$ about the enhancement of fluctuations in the vicinity of CEP, but the signals of two QCD PTs discussed above evidence either of the 3CEP existence [12] at which the CSR and deconfinement PTs are matched, or of existence of two CEPs for these PTs. The latter situation is, however, unrealistic, since at low baryonic densities the CSR and deconfinement are matched in a cross-over. Hence, we believe that the collision energy $\sqrt{s_{N N}} \simeq 8.8 \mathrm{GeV}$ is the vicinity of 3CEP.

This value of the collision energy is supported by the estimate of the CEP temperature $T_{\text {cep }} \in[148.4 ; 157.4] \mathrm{MeV}$ obtained in [23] on the basis of exactly solvable model of QGP bags with surface tension. This range of $T_{c e p}$ values is shown in the left panel of Fig. 1 by the horizontal dashed lines. Apparently, the CFO cannot occur above $T_{c e p}$ and, hence, the CFO temperature of the 3CEP vicinity obeys the inequality $T_{C F O} \leq T_{c e p}$. But from the left panel of Fig. 1 it is clear that the collision energy range of the 3 CEP vicinity is $\sqrt{s_{N N}} \in[9 ; 17.3] \mathrm{GeV}$. Combining these values with the collision energy range for the deconfinement PT $\sqrt{s_{N N}} \in$ [8.8; 9.2] GeV obtained recently in $[12,15]$, one finds a narrow range of collision energy for the 3CEP vicinity $\sqrt{s_{N N}} \in[9 ; 9.2] \mathrm{GeV}$, which simultaneously agrees with the QCD inspired exactly solvable model and the experimental signals of deconfinement.

\section{Conclusions}

Here we discuss the MHRGM, the irregularities found at chemical freeze-out and possible signals of two QCD phase transitions. From the 3CEP temperature $T_{\text {cep }} \in[148.4 ; 157.4]$ $\mathrm{MeV}$ obtained earlier on the basis of exactly solvable model of QGP bags with surface tension we found the collision energy range of 3CEP as $\sqrt{s_{N N}} \in[9 ; 17.3] \mathrm{GeV}$. However, combining it with the collision energy range for the deconfinement PT $\sqrt{s_{N N}} \in[8.8 ; 9.2] \mathrm{GeV}$ found recently in $[12,15]$, we determine a narrow range of collision energy for the 3CEP vicinity $\sqrt{s_{N N}} \in[9 ; 9.2] \mathrm{GeV}$. Evidently, this range is consistent both with the QCD inspired model and experimental findings.

Comparing the equation of state of the "White Matter" model [5] with the one used to describe the CSR in hadronic matter, we have come to a conclusion that results of [5] and the associated irregularities found in $[4,6]$ can be considered as the first evidence for the CSR in hadronic matter. However, the oversimplified parameterization of hadronic phase used in [5] led to a larger value of the threshold energy.

Acknowledgments.The work of K.A.B., A.I.I., V.V.S., B.E.G., D.O.S. and G.M.Z. was supported by the grants launched by the Section of Nuclear Physics of National Academy of Sciences of Ukraine. V.V.S. thanks the Fundação para a Ciência e Tecnologia (FCT), Portugal, for the financial support through the Grant No. UID/FIS/00099/2013 to make research at the CENTRA, Instituto Superior Técnico, Universidade de Lisboa. The work of 
L.V.B. and E.E.Z. was supported by the Norwegian Research Council (NFR) under grant No. 255253/F50 - CERN Heavy Ion Theory. L.V.B. and K.A.B. thank the Norwegian Agency for International Cooperation and Quality Enhancement in Higher Education for financial support, grant 150400-212051-120000 «CPEA-LT-2016/10094 From Strong Interacting Matter to Dark Matter». D.B.B. is grateful to the COST Action CA15213 "THOR" for networking support and to the MEPhI Academic Excellence program grant No 02.a03.21.0005 for partial support. D.B.B. acknowledges a support from the Polish National Science Centre (NCN) under grant no. UMO-2014/13/B/ST9/02621. The work of A.V.T. was partially supported by the Ministry of Science and Education of the Russian Federation, grant No. 3.3380.2017/4.6, and by National Research Nuclear University "MEPhI" in the framework of the Russian Academic Excellence Project (contract no. 02.a03.21.0005, 27.08.2013). The work of A.I.I. was done within the project SA083P17 of Universidad de Salamanca launched by the Regional Government of Castilla y Leon and the European Regional Development Fund.

\section{References}

[1] L. Adamczyk et al., [STAR Collaboration], Phys. Rev. C 93, 021903 (2016)

[2] P. Senger, Eur. Phys. J. A 52, 217 (2016) and references therein

[3] P. Senger, Nucl. Phys. A 862-863, 139 (2011) and references therein

[4] M. Gazdzicki, Z. Phys. C 66, 659 (1995)

[5] M. Gazdzicki and M.I. Gorenstein, Acta Phys. Polon. B 30, 2705 (1999)

[6] M. I. Gorenstein, M. Gazdzicki and K. A. Bugaev, Phys. Lett. B 567, 175 (2003)

[7] K. A. Bugaev et al., Phys. Part. Nucl. Lett. 12, 238 (2015)

[8] K. A. Bugaev et al., Eur. Phys. J. A 52, 175 (2016)

[9] K. A. Bugaev et al., Eur. Phys. J. A 52, 227 (2016)

[10] W. Cassing, A. Palmese, P. Moreau and E. L. Bratkovskaya, Phys. Rev. C 93, 014902 (2016)

[11] A. Palmese et al., Phys. Rev. C 94, 044912 (2016)

[12] K. A. Bugaev et al., Phys. Part. Nucl. Lett. 15, 210 (2018)

[13] S. Kabana and P. Minkowski,Int. J. Mod. Phys. A 26, 3035 (2011) and arXiv:1001.0707 [hep-ph]

[14] K. J. Sun, L. W. Chen, C. M. Ko, and Z. Xu, Phys. Lett. B 774, 103 (2017)

[15] K. A. Bugaev et al., KnE Energ. Phys. 3, 313 (2018)

[16] D. R. Oliinychenko, K. A. Bugaev and A. S. Sorin, Ukr. J. Phys. 58, 211 (2013)

[17] K. A. Bugaev, D. R. Oliinychenko, A. S. Sorin and G. M. Zinovjev, Eur. Phys. J. A 49, 30 (2013)

[18] K. A. Bugaev et al., Europhys. Lett. 10422002 (2013) and references therein

[19] V. V. Sagun, Ukr. J. Phys. 59755 (2014)

[20] K. A. Bugaev et al., Nucl. Phys. A 970, 133 (2018)

[21] V. V. Sagun et al., Eur. Phys. J. A 54, 100 (2018)

[22] A. Andronic, P. Braun-Munzinger and J. Stachel Nucl. Phys. A 772, 167 (2006)

[23] K. A. Bugaev et al., Phys. Atom. Nucl. 75, 707 (2012)

[24] K. A. Bugaev, M. I. Gorenstein, B. Kämpfer and V. I. Zhdanov, Phys. Rev. D 40, 2903 (1989)

[25] K. A. Bugaev, M. I. Gorenstein and D. H. Rischke, Phys. Lett. B 255, 18 (1991)

[26] Sz. Borsanyi et al., JHEP 08, 053 (2012) and references therein

[27] K. J. Sun, L. W. Chen, C. M. Ko, J. Pu and Z. Xu, arXiv:1801.09382v1 [nucl-th] 